\title{
Synthesis and Columnar Organization of Partially Fluorinated Dehydrobenz[18]annulenes
}

\author{
Sumitra Karki, Lucas J. Karas, Xiqu Wang, Judy I. Wu and Ognjen Š. Miljanic* \\ Department of Chemistry, University of Houston, 3585 Cullen Boulevard, Houston, Texas 77204-5003, United States
}

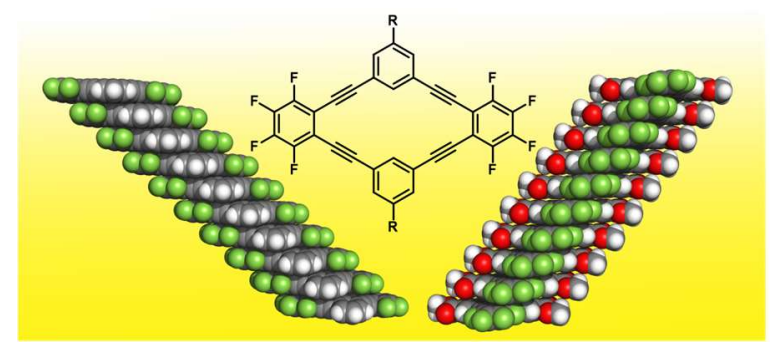

\begin{abstract}
Two diamond-shaped and partially fluorinated dehydrobenz[18]annulene macrocycles have been synthesized through a one-pot synthesis relying on fourfold Sonogashira coupling. Single crystal structures of the prepared macrocycles show continuous columnar stacks of these molecules that are mediated by the fluoroarene-alkyne, arene-alkyne, fluoroarene-fluoroarene, and alkyne-alkyne [ $\pi \cdots \pi]$ interactions instead of the expected fluoroarene-arene $[\pi \cdots \pi]$ interaction.
\end{abstract}

Shape-persistent dehydrobenzannulenes (DBAs; also known as arylene ethynylene macrocycles, AEMs) of various geometries and sizes have been pursued as synthetic targets for more than half a century. ${ }^{1}$ These $\pi$-conjugated macrocycles with conformationally rigid backbones and central cavities have been used in supramolecular chemistry and molecular recognition, ${ }^{2}$ host-guest chemistry, ${ }^{3}$ as components of organic sensors ${ }^{4}$ and organogels, ${ }^{5}$ nanoporous solids, ${ }^{6 \mathrm{a}, 6 \mathrm{~b}}$ and liquid crystals. ${ }^{6 \mathrm{c}}$ Moore and coworkers' report that these $\pi$-conjugated macrocycles can form dimers and higher aggregates through $[\pi \cdots \pi]$ interactions between the aromatic rings $^{7}$ started attempts to modulate this aggregation by the installation of electron-donating, ${ }^{8}$ electron-withdrawing, ${ }^{9}$ or hydrogen bonding ${ }^{10}$ units onto the DBAs. As benzene and hexafluorobenzene $[\pi \cdots \pi]$ stack in an electronically complementary and well-ordered alternating arrangement-a fact first recognized by Patrick and Prosser in 1960, ${ }^{11}$ and since studied in great detail ${ }^{12-}$ ${ }^{14}$ - we speculated that their joint incorporation into the DBA structures could result in the formation of higher order columnar assemblies in the solid state.

Given their interest to supramolecular chemistry, ${ }^{15}$ several partially and fully fluorinated DBAs have been synthesized and their aggregation behavior has been studied through concentrationdependent NMR and UV-vis spectroscopy, vapor pressure osmometry (VPO), and single crystal X-ray diffraction (XRD). ${ }^{10,16,17}$ Shu and co-workers have shown that the favored fluoroarene-arene interactions led to the aggregation of a hexagonally shaped, partially fluorinated diyne-linked DBAs, evidence by the SEM images which showed the formation of rod-like crystallites with a hexagonal crosssection. ${ }^{18}$ However, most of these studies were performed on the sixor three-fold symmetric DBAs in which the arene and the fluoroarene components shared either an ortho $^{16}$ or a meta ${ }^{17,18}$ substitution pattern. In this Letter, we present the synthesis and crystallographic characterization of two diamond-shaped partially fluorinated DBAs 1 and 2 (Scheme 1). These two macrocycles have two meta-connected arene and two ortho-connected tetrafluoroarene rings bridged by ethynylene moieties. Macrocycle 2 was synthesized to examine the influence of the exoannular substitution on the packing of the diamond-shaped macrocycle.

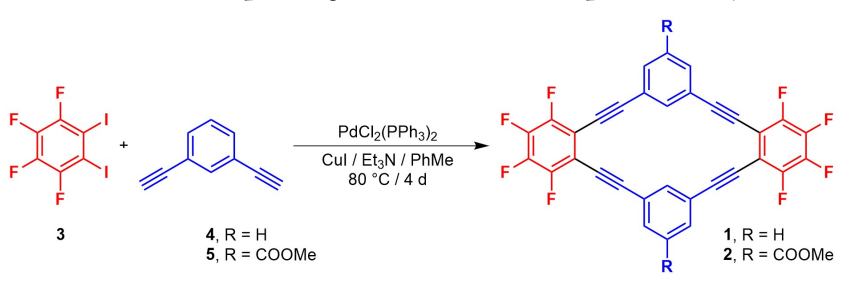

Scheme 1. Syntheses of dehydrobenz[18]annulene macrocycles 1 and 2 via Sonogashira coupling.

One-pot syntheses of macrocycles 1 and 2 (Scheme 1) were accomplished by reacting the commercially available 1,2-diiodo3,4,5,6-tetrafluorobenzene (3) with diynes $4^{19}$ and $5,{ }^{20}$ which themselves were prepared following literature procedures. Macrocycle 1 was isolated as a brown solid in $22 \%$ yield after successive recrystallizations from $\mathrm{PhMe} / \mathrm{Me}_{2} \mathrm{CO}$ and $\mathrm{PhMe} / \mathrm{MeCN}$ solvent systems. Macrocycle 2 was obtained after column chromatography eluting with DCM/hexanes as a white solid in $5 \%$ yield. Their relatively low yields were caused by the presence of side products which were either linear uncyclized oligomers or the reduced unreactive materials in which the arylappended iodine atom was replaced with a hydrogen. These side 
products were not isolated, but the one-step synthesis allowed the preparation of sufficient amounts of 1 and 2 .

To study the packing behavior of 1 and 2, single crystals of both macrocycles were grown. Diffraction-quality needle-shaped single crystals of 1 were produced by allowing the solution of 1 in hot $N, N$ diethylacetamide (DEA) to first slowly cool down to room temperature and then slowly evaporate over two days. Rod-shaped single crystals of 2 were obtained from its hot solution of THF, which was first slowly cooled to room temperature and then left to slowly evaporate over two days.

Macrocycle 1 crystalizes in the $P 2_{1} / \mathrm{n}$ space group with two molecules per unit cell. As shown in Figure 1A, it is only slightly distorted from planarity, with the dihedral angle between the planes of fluorinated and non-fluorinated rings of $9.58^{\circ}$, and the distance between two intraannular hydrogens of $2.42(3) \AA$. The extended packing diagram (Figure 1B) shows that the macrocycles organize into columnar stacks through slipped parallel stacking as shown in Figure 1C. Closer inspection of the packing shows that the crystal structure is held together by the T-shaped fluoroarene-fluoroarene interactions between the macrocycles of 1 with 2.91(3) A short contact between a fluorine and a carbon atom (Figure 1D). The interactions between the neighboring macrocycles within the stack are not the expected fluoroarene-arene interactions, but instead the apparent fluoroarene-alkyne $[\pi \cdots \pi]$ interactions highlighted in Figure 1E. Two macrocycles arrange in a slipped stacking interaction in a way where two of the carbon atoms of the fluoroarene reside above the alkyne with a centroid-centroid distance of 3.29(3) $\AA$, and two additional short arene-alkyne contacts of 3.37(3) Å; both are shorter than the sum of the van der Waals radii of two carbon atoms (3.40 ̊).

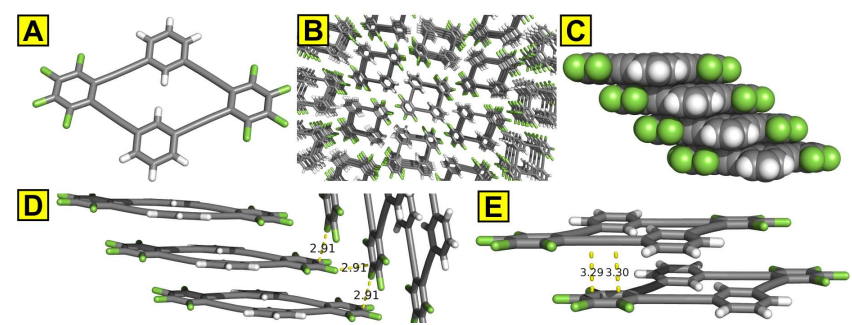

Figure 1. A: Crystal structure of macrocycle 1. Its extended packing diagram (B) shows slipped columnar stacking of molecules of 1 (C), meadiated by $\mathrm{T}$-shaped fluoroarene-fluoroarene interactions and (D) and fluoroarene-alkyne $[\pi \cdots \pi]$ interactions $(\mathrm{E})$.

Macrocycle 2 (Figure 2A) crystalizes in the $P 2_{1} / \mathrm{c}$ space group with two molecules per unit cell. The dihedral angle between the planes of fluorinated and non-fluorinated rings is again very small $\left(5.84^{\circ}\right)$ and the distance between the intraannular hydrogen atoms is 2.43(3) A. As seen in macrocycle 1, macrocycle 2 also forms a continuous columnar stack (Figure 2B) with slipped stacking between the macrocycles (Figure 2C) and T-shaped interactions (Figure 2D). However, their closer inspection reveals an interaction different from that observed in 1 : the molecules of 2 are not forming the stacking via the fluroarene-alkyne interaction but via fluroarenefluroarene and alkyne-alkyne interactions (Figure 2E). The two macrocycles are slipped in a way which results in the shortest contacts of 3.22(2) A between the carbons of the fluoroarenes, and 3.32(2) A between the carbons of alkynes. The third interaction between the two molecules of the macrocycle 2 is hydrogen bonding in the ester group with a short contact of 2.71(2) A between the oxygen and hydrogen of the methyl group. This $[\mathrm{C}-\mathrm{H} \cdots \mathrm{O}]$ hydrogen bond thus appears to play an important role in modulating the slipped-stacking of molecules of 2.

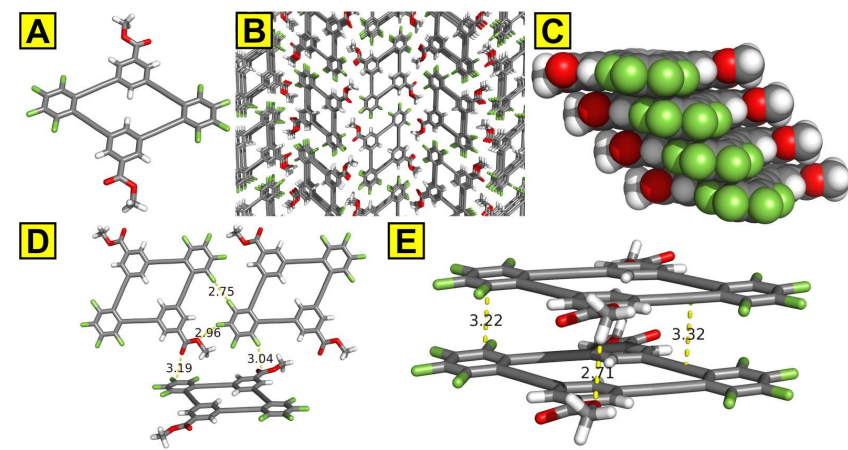

Figure 2. A: Crystal structure of macrocycle 2. Its extended packing diagram (B) shows slipped columnar stacking of molecules of 2 (C), mediated by T-shaped interactions (D), as well as a combination of hydrogen bonding, fluoroarene-fluoroarene, and alkyne-alkyne $[\pi \cdots \pi]$ interactions $(\mathrm{E})$.

The non-fluorinated analog of 1 , dehydrobenz [18] annulene 6, was previously synthesized. ${ }^{21}$ While the two compounds and the esterified analog 2 have very similar molecular structures, their packing arrangements differ quite profoundly. The crystal structure of 6 is held together by four T-shaped arene interactions between a hydrogen and a carbon of another arene (Figure 3A) with only short contact of 2.87(3) A, whereas the fluorinated analogs 1 and 2 form slipped parallel stacks via fluoroarene-alkyne and arene-alkyne $[\pi \cdots \pi]$ interactions in the case of 1 (Figure 3B), and hydrogen bonding, fluoroarene-fluoroarene and alkyne-alkyne $[\pi \cdots \pi]$ interactions in the case of 2 (Figure $3 \mathrm{C}$ ). The fluorination thus plays a critical role in forcing columnar assembly of macrocycles 1 and 2.
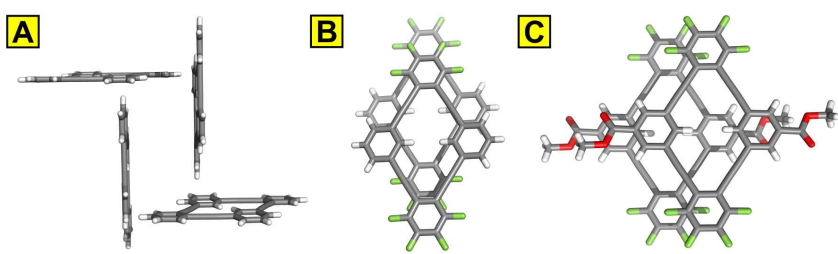

Figure 3. Comparison of the interaction patterns between the DBA macrocycles 6 (A), ${ }^{21} 1$ (B), and 2 (C).

Intrigued by the observation of fluoroarene-alkyne short contacts instead of the expected fluoroarene-arene $[\pi \cdots \pi]$ interactions in the case of 1 , we searched for other examples of fluoroarene-alkyne interactions in the Cambridge Structural Database (CSD). Figure 4 shows several examples. In the co-crystal of octafluoronaphthalene and diphenylacetylene (Figure 4A), alternate stacks form between two molecules via the fluoroarene-alkyne interactions with short contacts of $3.32(2) \AA .^{22}$ Similarly, as shown in Figure $4 \mathrm{~B}$, the structure of the co-crystal of 4,4-diphenylethynyl-2,2-bipyridine with hexafluorobenzene also shows that the fluoroarene is not interacting with the benzene ring but with the acetylenic moiety. ${ }^{23}$ Fluorinated bistolane derivate shown in Figure 4C also shows continuous stacking led by the $\pi$-interaction between a fluoroarene and an alkyne instead of the fluoroarene-arene interactions. ${ }^{24}$ Several additional examples show the presence of the fluroarenealkyne interaction despite the presence of an arene moiety available for a possible fluoroarene-arene interaction. ${ }^{25-28}$ This situation is observed in organic compounds, as well as in some of the metal complexes, although not many studies aimed to explain the observed patterns. ${ }^{29-33}$ 


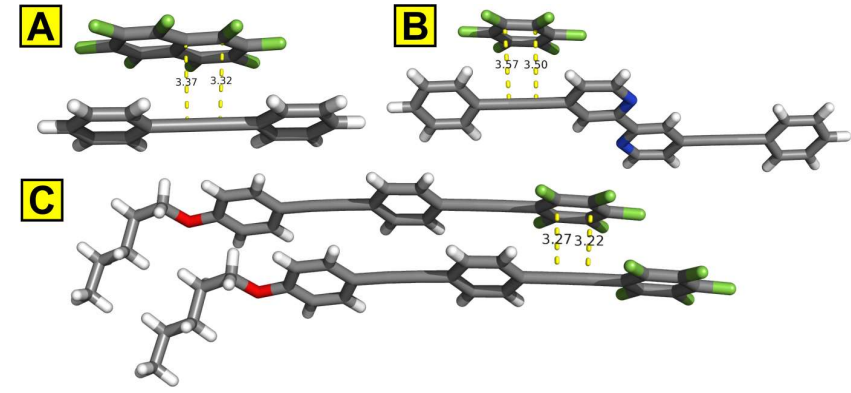

Figure 4. Selected structures from the CSD showing examples of fluoroarene-alkyne $[\pi \cdots \pi]$ interactions. ${ }^{22-24}$

The preference for the fluoroarene-alkyne interaction over the fluoroarene-arene interaction has also been observed in macrocyclic structures. For example, Bunz and co-workers observed that the cocrystal of hexafluorobenzene and the cyclic dimer of orthodiethynylbenzene shows a stacking pattern in which hexafluorobenzene centers above the alkyne moieties rather than the benzene rings of the macrocycle. ${ }^{34}$ Tahara and co-workers have synthesized the functionalized triangular dehydrobenzo[12]annulenes and observed that the fully fluorinated macrocycle stacks via fluoroarene-alkyne interactions. The same fluorinated DBA showed the fluoroarene-arene interaction when co-crystalized with the triply $N, N$-dimethylamino-substituted dehydrobenz[12]annulene. ${ }^{35}$

Moore and coworkers have concluded that the $\pi$-interactions between arenes and alkynes are not significantly stabilizing in DBAs. $^{7 a}$ However, fluorinating the arene may change this conclusion; the above-mentioned linear synthons, macrocycles, and our macrocycle 1 show that the fluoroarenes prefer to engage into the $[\pi \cdots \pi]$ interaction with the alkyne moieties even in the presence of arenes within the molecule available for a potential fluoroarenearene interaction.

Density functional theory (DFT) computations were performed for dimers of the macrocycle 1 and 2 to understand factors relevant to their self-association. Both $1\left(-31.4 \mathrm{kcal} \mathrm{mol}^{-1}\right)$ and $2(-39.6$ $\mathrm{kcal} / \mathrm{mol}^{-1}$ ) exhibit large negative dimerization energies, suggesting strong interactions between the macrocycles. All computations were performed at B3LYP-D3/6-311+G(d,p)//B3LYP-D3/6-31+G(d) employing Gaussian $16 .{ }^{36}$ The interaction energy for the dimer of 1 may be attributed to multiple $\pi$-interactions between the triple bonds and the arene rings. It is interesting to note that in the optimized geometry for alkyne-arene $\pi$-interactions, the alkyne normally resides over the center of the arene ring, but in the fluorinated macrocycle 1 the alkyne moieties reside over the edge of the fluoroarene rings. We hypothesize that this slipped packing helps maximize the number of $\pi$-interactions. The stronger interaction energy in the dimer of macrocycle 2 shows that hydrogen bonding plays an important role in stabilizing the packing arrangement. In fact, the computed interaction energy of macrocycle 2 is very close to that reported by Zhong et al. for stacked hexameric macrocycles with multiple hydrogen bonding interactions that drive the dimer formation. ${ }^{10}$ Thus, hydrogen bonding can be seen as the decisive factor in the difference in the packing behavior of the macrocycles 2 and 1 .

In summary, we prepared two partially fluorinated dehydrobenz[18] annules 1 and 2 via a one-pot route. Their single crystal structures show that the two macrocycles organize into columnar stacks, which are critically enabled by the presence of the fluorinated rings. In macrocycle 1, those rings engage in apparent $[\pi \cdots \pi]$ stacking fluoroarene-alkyne interactions. While the fluoroarene-arene interaction was found to direct the columnar stacks in the most symmetric hexameric macrocycles, in the case of diamond-shaped macrocycles with ortho- and meta-linked fluoroarene and arene groups, the columnar stacking has been achieved via fluoroarene-alkyne interaction. Our ongoing work aims to explore the generality of this assembly pattern and its relevance in the construction of advanced materials.

\section{ASSOCIATED CONTENT}

\section{Supporting Information}

Experimental procedures, copies of NMR spectra, spectroscopic analysis, and computational analysis at B3LYP-D3/6-31+G(d) (PDF). The Supporting Information is available free of charge on the ACS Publications website. Crystallographic data has been deposited with CCDC under access codes 2109154 and 2109155. These data can be obtained free of charge via www.ccdc.cam.ac.uk/data_request/ cif, or by emailing data_request@ccdc.cam.ac.uk, or by contacting The Cambridge Crystallographic Data Centre, 12 Union Road, Cambridge CB2 1EZ, UK; fax: +44 1223336033

\section{AUTHOR INFORMATION}

\section{Corresponding Author}

Ognjen Š. Miljanić, Department of Chemistry, University of Houston, 3585 Cullen Boulevard, Houston, Texas 77204-5003, United States; Email: miljanic@uh.edu.

\section{Author Contributions}

S. K. prepared and crystallized 1 and 2. X. W. solved their crystal structures. L. J. K. performed the computations with insights from J. I. W. S. K. and O. S. M. wrote the manuscript, incorporating comments from all other authors. All authors have given approval to the final version of the manuscript.

\section{ACKNOWLEDGMENT}

We acknowledge the generous financial support from the National Science Foundation (awards DMR-1904998 to O.Š.M. and CHE1751370 to J.I.W.), and the National Institute of General Medical Sciences of the National Institute of Health (grant R35GM133548 to J.I.W.). We acknowledge the use of the Sabine cluster and support from the Research Computing Data Core at the University of Houston.

\section{REFERENCES}

1. (a) Xu, Y.; von Delius, M. The Supramolecular Chemistry of Strained Carbon Nanohoops. Angew. Chem. Int.Ed.2020, 59, 559-573; (b) Toyota, S.; Tsurumaki, E. Exploration of Nano-Saturns: A Spectacular Sphere-Ring Supramolecular System. Chem. Eur.J. 2019, 25, 6878-6890; (c) Smith, M. S.; Miljanić, O. Š. Arylene Ethynylene Macrocycles: from Molecular Hosts to Components of High-performance Supramolecular Architectures. Org. Biomol. Chem. 2015, 13, 7841-7845; (d) Zhang, W.; Moore, J. S. ShapePersistent Macrocycles: Structures and Synthetic Approaches from Arylene and Ethynylene Building Blocks. Angew. Chem. Int. Ed. 2006, 45, 44164439; (e) Höger, S. Shape-Persistent Phenylene-Acetylene Macrocycles: Large Rings-Low Yield? Angew. Chem. Int. Ed. 2005, 44, 3806-3808; (f) Zhao, D.; Moore, J. S. Shape-Persistent Arylene Ethynylene Macrocycles: Syntheses and Supramolecular Chemistry. Chem. Commun. 2003, 807818.

2. (a) Iyoda, M.; Yamakawa, J.; Rahman, M. J. Conjugated Macrocycles: Concepts and Applications. Angew. Chem. Int. Ed.2011, 50, 10522-10553; 
(b) Höger, S. Shape Persistent Rings and Wheels. Pure Appl. Chem. 2010, 82, 821-830; (c) Yamaguchi, Y.; Yoshida, Z.-i. Shape-Persistency and Molecular Function in Heteromacrocycles: Creation of Heteroarenecyclynes and Arene-Azaarenecyclynes. Chem. Eur. J. 2003, 9, 5430-5440; (d) Haley, M. M.; Pak, J. J.; Brand, S. C. Macrocyclic Oligo(Phenylacetylenes) and Oligo(Phenyldiacetylenes). Top. Curr. Chem. 1999, 201, 81-130; (e) Moore, J. S. Shape-Persistent Molecular Architectures of Nanoscale Dimension. Acc. Chem. Res. 1997, 30, 402-413. 3. Popov, I.; Chen, T.-H.; Belyakov, S.; Daugulis, O.; Wheeler, S. E.; Miljanić, O. Š. Macrocycle Embrace: Encapsulation of Fluoroarenes by mPhenylene Ethynylene Host. Chem. Eur. J. 2015, 21, 2750-2754.

4. Gross, D. E.; Zang, L.; Moore, J. S. Arylene-Ethynylene Macrocycles: Privileged Shape-Persistent Building Blocks for Organic Materials. Pure Appl. Chem. 2012, 84, 869-878.

5. Ruan, Y.; Li, Q.-H.; Shu, L.; Wan, J.-H. A Shape-Persistent Arylene Ethynylene Macrocycle with a Multiple Acetamide Modified Cavity: Synthesis and Gelation. Soft Matter 2021, 17, 3242-3249.

6. (a) Chen, T.-H.; Popov, I.; Chuang, Y.-C.; Chen, Y.-S.; Miljanić, O. Š. A Mesoporous Metal-Organic Framework Based on a Shape-Persistent Macrocycle. Chem. Commun. 2015, 51, 6340-6342; (b) Grave, C.; Schlüter, A. D. Shape-persistent, Nano-Sized Macrocycles. Eur. J. Org. Chem. 2002, 18, 3075-3098; (c) Höger. S. Shape-Persistent Macrocycles: From Molecules to Materials. Chem. Eur.J. 2004, 10, 1320-1329.

7. (a) Shetty, A. S.; Zhang, J.; Moore, J. S. Aromatic $\pi$-Stacking in Solution as Revealed through the Aggregation of Phenylacetylene Macrocycles. $J$. Am. Chem. Soc. 1996, 118, 1019-1027: (b) Zhang, J.; Moore, J. S. Aggregation of Hexa(phenylacetylene) Macrocycles in Solution: A Model System for Studying $\pi-\pi$ Interactions. J. Am. Chem. Soc. 1992, 114, $9701-$ 9702.

8. Höger. S.; Bonrad, K.; Mourran, A.; Beginn, U.; Möeller, M. Synthesis, Aggregation, and Adsorption Phenomena of Shape-Persistent Macrocycles with Extraannular Polyalkyl Substituents. J. Am. Chem. Soc. 2001, 123, 5651-5659.

9. Tobe, Y.; Utsumi, N.; Nagano, A.; Naemura, K. Synthesis and Association Behavior of [4.4.4.4.4.4] Metacyclophanedodecayne Derivatives with Interior Binding Groups. Angew. Chem. Int. Ed. 1998, 37, 1285-1287.

10. Zhong, Y.; Yang, Y.; Shen, Y.; Xu, W.; Wang, Q.; Connor, A. L.; Zhou, X.; He, L.; Zeng, X.C.; Shao, Z.; Lu, Z-1.; Gong, B. Enforced Tubular Assembly of Electronically Different Hexakis( $m$-Phenylene Ethynylene) Macrocycles: Persistent Columnar Stacking Driven by Multiple HydrogenBonding Interactions. J. Am. Chem. Soc. 2017, 139, 15950-15957.

11. Patrick, C. R.; Prosser, G. S. A Molecular Complex of Benzene and Hexafluorobenzene. Nature 1960, 187, 1021.

12. Williams, J. H. Modeling the Vibrational Dynamics of Solid Benzene: Hexafluorobenzene. The Anatomy of a Phase Transition. Chem. Phys. 1993, 172, 171-186.

13. Tsuzuki, S.; Uchimaru, T.; Mikami, M. Intermolecular Interaction between Hexafluorobenzene and Benzene: Ab Initio Calculations Including $\operatorname{CCSD}(\mathrm{T})$ Level Electron Correlation Correction. J. Phys. Chem. A. 2006, 110, 2027-2033.

14. Bhandary, S.; Chopra, D. Assessing the Significance of Hexafluorobenzene as a Unique Guest Agent through Stacking Interactions in Substituted Ethynylphenyl Benzamides. Cryst. Growth. Des. 2018, 18, 3027-3036.

15. (a) Nieland, E.; Weingart, O.; Schmidt, B. M. Fluorinated Azobenzenes as Supramolecular Halogen-Bonding Building Blocks. Beilstein J. Org. Chem. 2019, 15, 2013-2019; (b)Yi, W.-B.; Ma, J.-J.; Jiang, L.-Q.; Cai, C.; Zhang, W. Synthesis and Uses of Fluorous and Highly Fluorinated Macrocyclic and Spherical Molecules. J. Fluor. Chem. 2014, 157, 84-105; (c) Chopra, D.; Row, T. N. G. Role of Organic Fluorine in Crystal Engineering. Cryst. Eng. Comm. 2011, 13, 2175-2186; (d) Hird, M. Fluorinated Liquid Crystals - Properties and Applications. Chem. Soc. Rev. 2007, 36, 2070-2095; (e) Plenio, H. Partially Fluorinated Macrocycles: Synthesis of the Tetrafluoro Analogue of the [2S.20.20]-Cryptand and the Crystal Structure of the Sodium Complex. Inorg. Chem. 1994, 33, 61236127; ( $f)$ Farnham, W. B.; Roe, D. C.; Dixon, D. A.; Calabrese, J. C.; Harlow, R. L. Fluorinated Macrocyclic Ethers as Fluoride Ion Hosts. Novel
Structures and Dynamic Properties. J. Am. Chem. Soc. 1990, 112, 77077718.

16. Iyoda, M.; Vorasingha, A.; Kuwatani, Y.; Yoshida, M. A One-Step Synthesis of Dehydro[12] annulenes using Palladium-Catalyzed Reaction of $o$-diiodoarenes with Acetylene Gas. Tetrahedron Lett. 1998, 39, 47014704.

17. Shu, L.; Mayor, M. Shape-Persistent Macrocycle with a Selfcomplementary Recognition Pattern Based on Diacetylene-linked Alternating Hexylbenzene and Perfluorobenzene Rings. Chem. Commun. 2006, 39, 4134-4136.

18. Shu, L.; Müri, M.; Krupke, R.; Mayor, M. Shape-Persistent Macrocycles Comprising Perfluorinated Benzene Subunits: Synthesis, Aggregation Behaviour and Unexpected $\mu$-Rod Formation. Org. Biomol. Chem. 2009, 7, 1081-1092.

19. Neenan, T. X.; Whitesides, G. M. Synthesis of High Carbon Materials from Acetylenic Precursor. Preparations of Aromatic Monomers Bearing Multiple Ethynyl Groups. J. Org. Chem. 1988, 55, 2489-2496.

20. (a) Wang, S.; Liu, L.; Guo, X.; Li, G.; Wang, X.; Dong, H.; Li, Y.; Zhao, W. Synthesis of Novel Natural Product-like Diaryl Acetylenes as Hypoxia Inducible Factor-1 Inhibitors and Antiproliferative Agents. RSC Adv. 2019, 9, 13878-13886; (b) Bose, P.; Bai, L.; Ganguly, R.; Zou, R.; Zhao, Y. Rational Design and Synthesis of a Highly Porous Copper-Based Interpenetrated Metal-Organic Framework for High $\mathrm{CO}_{2}$ and $\mathrm{H}_{2}$ Adsorption. Chem. Plus. Chem. 2015, 80, 1259-1266.

21. Miljanić, O. Š.; Vollhardt, K. P. C.; Whitener, G. D. An Alkyne Metathesis-Based Route to ortho-Dehydrobenzannulenes. Synlett 2003, 0029-0034.

22. Collings, J. C.; Batsanov, A. S.; Howard, J. A. K.; Marder, T. B. Octafluoronaphthalene-diphenylacetylene (1/1). Acta. Cryst. C: Cryst Struct. Comm. 2001, 57, 870-872.

23. Hori, A.; Takatani, S.; Miyamoto, T. K.; Hasegawa, M. Luminescence from $\pi-\pi$ Stacked Bipyridines through Arene-Perfluoroarene Interactions. Cryst. Eng. Comm. 2009, 11, 567-569.

24. Yamada, S.; Miyano, K.; Konno, T.; Agou, T.; Kubota, T.; Hosokai, T. Fluorine-Containing Bistolanes as Light-Emitting Liquid Crystalline Molecules. Org. Biomol. Chem. 2017, 15, 5949-5958.

25. Clyburne, J. A. C.; Hamilton, T.; Jenkins, H. A. The Molecular Quadrupole Moment: Solid State Architectures Containing Organic and Organometallic Molecules. Cryst. Eng. 2001, 4, 1-9.

26. (a) Yamada, S.; Miyano, K.; Agou, T.; Kubota, T.; Konno. T. 2Chloroalkoxy-Substituted Pentafluorinated Bistolanes as Novel LightEmitting Liquid Crystals. Crystals 2019, 9, 195; (b) Yamada, S.; Morita, M.; Agou, T.; Kubota, T.; Ichikawa, T.; Konno, T. Thermoresponsive Luminescence Properties of Polyfluorinated Bistolane-type Light-Emitting Liquid Crystals. Org. Biomol. Chem 2018, 16, 5609-5617.

27. Collings, J. C.; Burke, J. M.; Smith, P. S.; Batsanov, A. S.; Howard, J. A. K.; Marder, T. B. The Synthesis and Crystal Structures of Halogenated Tolans $p-\mathrm{X}-\mathrm{C}_{6} \mathrm{H}_{4}-\mathrm{C} \equiv \mathrm{C}-\mathrm{C}_{6} \mathrm{~F}_{5}$ and $p-\mathrm{X}-\mathrm{C}_{6} \mathrm{~F}_{4}-\mathrm{C} \equiv \mathrm{C}-\mathrm{C}_{6} \mathrm{H}_{5}(\mathrm{X}=\mathrm{F}, \mathrm{Cl}, \mathrm{Br}, \mathrm{I})$. Org. Biomol. Chem. 2004, 2, 3172-3178.

28. Stein, M.; Berger, R.; Seichter, W.; Hulliger, J.; Weber, E. Specific Interaction Modes in the Crystal Structures of Oligofluorinated Tolanes Featuring Additional Electron Donor and Acceptor Groups. J. Fluor. Chem. 2012, 135, 231-239.

29. Melen, R. L.; Hansmann, M. M.; Lough, A. J.; Hashmi, A. S. K.; Stephan, D. W. Cyclisation versus 1,1-Carboboration: Reactions of $\mathrm{B}\left(\mathrm{C}_{6} \mathrm{~F}_{5}\right)_{3}$ with Propargyl Amides. Chem. Eur.J. 2013, 19, 11928-11938.

30. Gunay, A.; Jones, W. D. Cleavage of Carbon-Carbon Bonds of Diphenylacetylene and Its Derivatives via Photolysis of Pt Complexes: Tuning the $\mathrm{C}-\mathrm{C}$ Bond Formation Energy toward Selective $\mathrm{C}-\mathrm{C}$ Bond Activation. J. Am. Chem. Soc. 2007, 129, 8729-8735.

31. Itoh, T.; Kondo, M.; Kanaike, M.; Masaoka, S. Arene-Perfluoroarene Interactions for Crystal Engineering of Metal Complexes: Controlled SelfAssembly of Paddle-Wheel Dimers. CrystEngComm. 2013, 15, 6122-6126. 32. Walter, V.; Gao, Y.; Grzegorzek, N.; Krempe, M.; Hampel, F.; Jux, N.; Tykwinski, R. R. Building from Ga-Porphyrins: Synthesis of Ga-Acetylide Complexes Using Acetylenes and Polyynes. Angew. Chem. Int. Ed. 2019, $58,494-498$. 
33. Johnson, A.; Gimeno, M. C. Synthesis of Propargyl-Functionalized NHC Gold Complexes. Organometallics 2017, 36, 1278-1286.

34. Bunz, U. H. F.; Enkelmann, V. Structure Elucidation, Packing, and SolidState Behavior of the Eglinton-Galbraith Dimer. Chem. Eur. J. 1999, 5, 263-266.

35. Tahara, K.; Fujita, T.; Sonoda, M.; Shiro, M.; Tobe, Y. Donors and Acceptors Based on Triangular Dehydrobenzo[12] annulenes: Formation of a Triple-Layered Rosette Structure by a Charge-Transfer Complex. J. Am. Chem. Soc. 2008, 130, 14339-14345.

36. Frisch, M.J.; Trucks, G. W.; Schlegel, H. B.; Scuseria, G. E.; Robb, M. A.; Cheeseman, J. R.; Scalmani, G.; Barone, V.; Petersson, G. A.; Nakatsuji, H.; Li, X.; Caricato, M.; Marenich, A. V.; Bloino, J.; Janesko, B. G.; Gomperts, R.; Mennucci, B.; Hratchian, H. P.; Ortiz, J. V.; Izmaylov, A. F.; Sonnenberg, J. L.; Williams-Young, D.; Ding, F.; Lipparini, F.; Egidi, F.; Goings, J.; Peng, B.; Petrone, A.; Henderson, T.; Ranasinghe, D.; Zakrzewski, V. G.; Gao, J.; Rega, N.; Zheng, G.; Liang, W.; Hada, M.; Ehara, M.; Toyota, K.; Fukuda, R.; Hasegawa, J.; Ishida, M.; Nakajima, T.; Honda, Y.; Kitao, O.; Nakai, H.; Vreven, T.; Throssell, K.; Montgomery, J. A., Jr.; Peralta, J. E.; Ogliaro, F.; Bearpark, M. J.; Heyd, J. J.; Brothers, E. N.; Kudin, K. N.; Staroverov, V. N.; Keith, T. A.; Kobayashi, R.; Normand, J.; Raghavachari, K.; Rendell, A. P.; Burant, J. C.; Iyengar, S. S.; Tomasi, J.; Cossi, M.; Millam, J. M.; Klene, M.; Adamo, C.; Cammi, R.; Ochterski, J. W.; Martin, R. L.; Morokuma, K.; Farkas, O.; Foresman, J. B.; Fox, D. J. Gaussian 16, Revision A.03 (Gaussian, Inc., Wallingford CT, 2016). 